\title{
Some Properties of the General n-Trailer
}

\author{
Claudio Altafini* \\ Optimization and Systems Theory \\ Royal Institute of Technology \\ SE-10044, Stockholm, Sweden \\ e-mail: altafini@math.kth.se \\ tel: $\quad+4687907507$ \\ fax: $\quad+468225320$
}

July 19,2000

\begin{abstract}
If some of the trailers of the so-called $n$-trailer system are connected via a kingpin hitch, the kinematic model of the system is more complicated that in the standard case. However, something can still be said about the structural properties of the system, like controllability. The more complicated equations can be interpreted in terms of virtual steering wheels placed on the off-axle joints with steering angle which is a nonlinear feedback from the original configuration state. Quite remarkably, the extra singularities of the system have an explanation in terms of these virtual steering wheels. This is also sufficient to assert that the general $n$-trailer problem can be embedded into the corresponding multisteering $n$-trailer system. The multi-chained form available for this last system can be recovered also for the general $n$-trailer if we replace the extra steering inputs with the aforementioned feedback loops.
\end{abstract}

Keywords: Mobile robots, Nonholonomic Constraints, Nonlinear Systems, Pfaffian Systems, Controllability, Singularity, Embedded System, Chained form.

\section{Introduction}

The $n$-trailer system, i.e. a mobile robot pulling an arbitrary number of trailers is a typical example of system in which the nonholonomic kinematic constraints, due to the assumption of rolling without slipping of the wheels, play a determinant role. The configuration of the system is given by two position coordinates and $n+1$ angles and, normally, two are the

${ }^{*}$ This work was supported by the Swedish Foundation for Strategic Research through the Center for Autonomous Systems at KTH 
available inputs, representing the steering and translational actuators of the pulling cart, even though systems with more steering inputs have been studied in [Bushnell et al. 1993] and [Tilbury et al. 1995]. The number of trailers can be increased at will: adding a trailer simply means adding a nonholonomic constraint to the system or, equivalently, an orientation angle to the configuration space.

The usual model for the pulling cart and for the trailers consists of a single axle with two wheels that are assumed parallel, neglecting, in the steering pair, the difference due to the fact that rotations must occur around a single center of rotation, see [Alexander and Maddox 1988]. Each axle is hitched to the preceding trailer by means of a rigid bar. In the case extensively treated in the literature, starting from Laumond [Laumond 1990], every axle is hitched exactly in the middle of the axle in front of it, i.e. only axle-to-axle connection are present. We will call this the standard n-trailer problem. A rich literature has grown for the standard $n$-trailer problem dealing with controllability [Laumond 1990], open loop motion generation [Murray and Sastry 1993], [Rouchon et al. 1993], closed loop control strategies [Bloch et al. 1992], [Sørdalen 1993b] and many others (see [Kolmanovsky and McClamroch 1995] for a survey) for different control problems like point stabilization, point tracking or path following. The peculiarity of this kind of system is that its nonholonomic structure, which 'permits' controllability even if the system is underactuated, is directly visible in the model formulation as a collection of nonholonomic constraints on the velocity. In geometric terms, the $n$-trailer can be seen as an higher order contact manifold whose defining functions are the set of non-integrable one forms corresponding to the velocity constraints on the wheels. In particular, it was shown in [Sørdalen 1993a] and [Tilbury et al. 1995] that the resulting system can be converted into a particular canonical form, called chained form for which the handling of the above mentioned problems result simplified. The chained form was first introduced by [Murray and Sastry 1993] as a particular class of equations for driftless systems. Although nonlinear, it has an underlying linear structure, reminiscent of the Brunovsky normal form. Since it was introduced, the chained form has been extensively used to treat all of the relevant control problems of the nonholonomic systems, see [Samson 1995], [Sørdalen and Egeland 1995] for instance. In [Tilbury et al. 1995], a connection was made with its dual, the so-called Goursat normal form in the language of the exterior differential systems. The 2-input chained form was extended to multi chained form (intended as multi-input single generator chained form) in [Tilbury et al. 1995], to take into account more complex systems with more that one steering input. An application of 3 -input chained form was the fire-truck example presented in [Bushnell et al. 1993].

In many practical situations, the standard $n$-trailer is not a realistic configuration: in fact, to improve maneuverability or due to the cumbersome shape of the trailer body, it happens that a trailer can be attached not exactly in the middle of the preceding axle but at a positive distance from it. When one or more trailers present off-hitching, we will name the system general $n$-trailer. Examples of such systems are the truck and trailers that we normally see on our highways or a real car pulling a trailer, or special articulated vehicles, like the so-called LHD (Load-Haul-Dump) a two-axle truck used for mining applications, [Altafini 1999].

Including kingpin hitches into the kinematics of the $n$-trailer results into an extra number of terms that need to be added to the basic dynamic equations. This suggests that some of the 
techniques for the standard $n$-trailer can be generalized to our system. In particular, results on controllability obtained by [Laumond 1990, Murray and Sastry 1993] can be extended quite directly. We will check local controllability in two different ways: first via the rank of the control Lie algebra as is normally done; then in the dual way, calculating the derivative flag of the Pfaffian system which is obtained from the dual representation of the general $n$-trailer. The comparison of the two procedures is quite interesting: as is often the case, the local maximal nonintegrability condition is much easier to check on the dual system.

The above mentioned extra terms in the kinematics corresponds to irregularities that 'break the chain' that constitute the 'backbone' of the $n$-trailer system so that the global 2input chained form is lost. We will show, however, that this breaking points (the off-hitching joints) can be substituted by 'virtual' passive steering wheels whose steering angle is uniquely determined by the configuration state by means of nonlinear feedback. Each virtual steering wheel corresponds to an internal feedback loop from the configuration state of the system. Opening all these feedback loops, we have a multisteering $n$-trailer system for which a multiinput chained form exists [Tilbury et al. 1995]. All the chains admit the same generator and this generator is the 'classical' one used for the 2-input chained form for the standard $n$-trailer. Since the general $n$ trailer has only two degrees of freedom (i.e. two inputs), the feedback of the virtual steering wheels will take the place of the exogenous input used in [Tilbury et al. 1995] and will link two consecutive chains. This is equivalent to consider only a particular submanifold of the multisteering $n$-trailer system and it corresponds to think of the general $n$-trailer as an embedding into the multisteering $n$-trailer. Moreover, our chained form results simplified with respect to [Tilbury et al. 1995] in the sense that no dynamic extension of the system is needed.

The more complex nature of the system reflects in an higher number of singularities when compared to the standard case, see [Jean 1996]. We give a physical interpretation of these extra singularities in terms of the steering angles of the above mentioned virtual wheels.

Whenever possible, we carry out explicit calculations for the cartesian frame we choose. In fact, one of the main advantages of the $n$-trailer configuration is that it has a state space description which is simple enough to allow writing down and manipulate exact formulas in the original coordinate setting for both the primal and the dual representations of the system, even for a generic number of trailers. The resulting formulae we get are somewhat lengthy, but we do not think that this is obscuring their geometric properties.

\section{Mathematical preliminaries}

The material in the present Section is largely taken from standard textbooks in Nonlinear Control like [Isirori 1995, Nijmeijer and van der Shaft 1990] and from [Abraham et al. 1983, Bryant et al. 1991] for the basic facts about exterior systems and differential forms. A more thorough presentation of this material in the same context of Mobile Robotics can also be found in [Pappas et al. 1998, Tilbury et al. 1995]. 


\subsection{Underactuated drift-free nonlinear systems}

Definition 1 An underactuated drift-free control-affine nonlinear control system is a collection of $r$ differential equations in the variables $x$ and $u_{i}, i=1, \ldots r$

$$
\dot{x}=\sum_{i=1}^{r} g_{i}(x) u_{i}
$$

where $x \in \mathcal{D} \subset \mathbb{R}^{q}$ with $q>r$ and $g_{i}$ are input vector fields $g_{i}: \mathcal{D} \rightarrow \mathbb{R}^{q}$. We assume that $\mathcal{D}$ contains the point $x_{0}$ of $\mathbb{R}^{q}$.

The expression $(1)$ can be intended as a representation in a local cover $\left(\mathcal{D}, x=\left(x_{1}, \ldots x_{q}\right)\right)$ of a point $p$ leaving on an abstract manifold $M$. The tangent space at $p \in M$ is indicated with $T_{p} M$ and its expression in the local coordinate chart $x$ as $T_{x} M$. The tangent space $T_{p} M$ has the same dimension of the manifold $M$.

Definition 2 The distribution $\Delta$ associated with the control system (1) is a collection of independent vector fields $g_{i}$. On each point $x \in \mathcal{D}$ the distribution $\Delta$ gives a vector subspace of $T_{x} M$ :

$$
\Delta(x)=\operatorname{span}\left\{g_{1}(x), \ldots g_{r}(x)\right\} \quad x \in \mathcal{D}
$$

We assume $g_{i}$ to be $C^{\infty}(\mathcal{D})$ and $x_{0}$ to be a regular point of $\Delta$ i.e. $\operatorname{dim} \Delta(x)=r \forall x \in \mathcal{D}$.

For the particular kind of system studied in this paper, the dual point of view of the distribution is particularly interesting because, as we will show below, it corresponds to highlight the nonholonomic constraints of the system. If we call $T_{p}^{*} M$ ( $T_{x}^{*} M$ in coordinates) the cotangent space, dual to $T_{p} M$, then we have:

Definition 3 A codistribution I associated with the control system (1) is a collection of $s=q-r$ smooth and linearly independent (over the ring of smooth functions) covector fields $\alpha^{j}$ that annihilate $\Delta$ on each point $x \in \mathcal{D}$ :

$$
\begin{aligned}
I(x) & =\operatorname{span}\left\{\alpha^{1}(x), \ldots, \alpha^{s}(x)\right\} \quad j=1, \ldots, s \\
& =\left\{\alpha^{j}(x) \in T_{x}^{*} M \text { s.t. }<\alpha^{j}(x), g_{i}(x)>=0 \quad \forall j=1, \ldots, s, i=1, \ldots, r\right\}
\end{aligned}
$$

We assume to work in a domain $\mathcal{D}$ in which the one-forms $\alpha^{j}(x)$ are $C^{\infty}$ sections of the exterior algebra over $T_{x}^{*} M$ and the codistribution $I$ is a smooth assignment (and therefore, at each point $x$, a vector subspace of $T_{x}^{*} M$ ) both with respect to the wedge product, i.e. the alternating (normalized) tensor product. What (3) says is that, in coordinates, the one-forms $\alpha^{j}(x)$ can be written as a $s \times r$ matrix such that the $g_{i}(x)$ constitute a basis for the right null space of this matrix. Then, if we want to be able to use the machinery of exterior differential systems, we have to endow the codistribution $I$ with some extra structure in order to make sure that the solution of our collection of one-forms is indeed an integrable distribution. This property correspond to the regularity assumption of a point in the distribution case. Such a special case of codistribution is called a Pfaffian system.

Definition 4 The codistribution formed by the smooth and independent one-forms $I=$ $\left\{\alpha^{1}, \ldots, \alpha^{s}\right\}$ is said a Pfaffian system if it generates an ideal $\mathcal{I}$ which is closed under exterior differentiation. 
The ideal generated by $I$ is

$$
\mathcal{I}=\left\{\alpha^{i} \wedge \theta \text { s.t. } \alpha^{i} \in I, \quad \theta \in \Omega(M)\right\}
$$

where $\Omega(M)$ is the module of smooth exterior differential forms of all orders on $M$.

\subsection{Local controllability for underactuated systems}

A fundamental (and well-studied) issue to deal with for underactuated systems is controllability, see [Nijmeijer and van der Shaft 1990] or, for example, the classical survey paper [Hermann and Krener 1977]. In what follows we are interested only in the local properties around a regular point $x_{0}$.

Definition 5 The system (1) is said small-time locally controllable at $x_{0} \in \mathcal{D}$ if we can reach nearby points in arbitrarily small amounts of time remaining in $\mathcal{D}$.

It is well-known that the notion of local controllability (which coincides with local strong accessibility for drift-free systems) can be checked in geometric terms by considering the span of the commutators of the vector fields that generate the system. This idea is strictly connected with that of involutive distribution via the Frobenius theorem that gives necessary and sufficient condition for (local) complete integrability of a distribution. This is essentially equivalent to say that the annihilator space of $\Delta$ has to be spanned by exact differentials, at least locally.

The fundamental tool to test local controllability is the Chow theorem which asserts that a system is locally controllable if and only if it is maximally nonintegrable.

The vector fields of $\Delta$, together with their commutators, form an algebra, called the control Lie algebra. In order to construct it, one has to build a filtration, patching together the vector fields of $\Delta$ and all the new independent commutators produced at each level of Lie bracketing $\Delta_{0}=\Delta, \Delta_{i}=\operatorname{span}\left\{\Delta_{i-1}+\left[\Delta_{i-1}, \Delta_{i-1}\right]\right\}$, such that

$$
\Delta_{0} \subset \Delta_{1} \subset \ldots \subset \Delta_{k}
$$

for some finite $k$. Different rules for building the above filtration are given in [Laumond 1993a, Laumond 1993b, Murray and Sastry 1993]. In a regular point, the dimension of the filtration (called the growth vector) stabilizes in correspondence of the control Lie algebra. We have local controllability when the rank of the control Lie algebra is equal to the dimension of the tangent space.

A dual characterization can be carried out for the Pfaffian system corresponding to (1). In particular, dually to the filtration (4), we can construct a descending chains of Pfaffian systems called derivative flag

$$
I^{(0)} \supset I^{(1)} \supset \ldots \supset I^{(k)}
$$

where $I^{(0)}=I$ and $I^{(j+1)}=\left\{\alpha^{i}=\in I^{(j)}\right.$ s.t. $\left.d \alpha^{i} \equiv 0 \bmod I^{(j)}\right\}$ is the derived Pfaffian system of $I^{(j)}$. The expression $d \alpha^{i} \equiv 0 \bmod I^{(j)}$ is called a congruence and means that the exterior derivative of $\alpha^{i}$ is a linear combination of the one-forms of $I^{(j)}$ (over the ideal $\mathcal{I}^{(j)}$ ), i.e. $d \alpha^{i} \wedge \alpha^{1_{j}} \wedge \ldots \wedge \alpha^{s_{j}}=0 \quad \forall \alpha^{l_{j}} \in I^{(j)}$. 
Similarly to the filtration, also the derivative flag stops at a certain $k$ for regular points. The maximally nonholonomy condition can therefore be restated in terms of the derivative flag, saying that local controllability is equivalent to the existence of an integer $k$ at which the derivative flag becomes empty: $I^{(k)}=0$.

To have controllability, the bottom system of the derivative flag, which is always integrable by the Frobenius theorem, has to be empty. This implies that there is no integrable subsystem of the original system, i.e. the solution trajectories of $I$ are not constraints to lie on a leave of a (nontrivial) foliation of $M$.

\subsection{Singularities}

Regularity of $x_{0}$ means that the distribution $\Delta$ does not loose rank in the neighborhood $\mathcal{D}$ of $x_{0}$. A similar condition is of interest for the filtration (4). If the dimension of the entire sequence (4) is constant in $\mathcal{D}$, then we call $x_{0}$ regular with respect to the filtration, in order to distinguish from the regularity with respect to $\Delta$ only.

Definition 6 A point $x_{0}$ which is not regular with respect to the filtration is said singular.

All the singular points of the system form the so-called singular locus of the system. The knowledge of the singular locus is important when checking controllability: in fact in correspondence of such a zero dimensional submanifold, the number of Lie bracketing operations needed to span the whole tangent space is different from the points which are regular with respect to the filtration. The complexity of such a check (which is proportional to the complexity of a steering algorithm for the system) obviously increases in the singular points.

\subsection{Embedding map}

The next concept we need is that of embedding map of a manifold.

Definition 7 Given two smooth manifolds $M_{1}$ and $M_{2}$ with $\operatorname{dim}\left(M_{1}\right)=q_{1}$ and $\operatorname{dim}\left(M_{2}\right)=$ $q_{2}, q_{1} \leq q_{2}$, the $C^{\infty}$ map $f: M_{1} \rightarrow M_{2}$ is called a local immersion of $x \in M_{1}$ if there exists a neighborhood $\mathcal{D} \in M_{1}$ of $x_{0}$ such that $\operatorname{rank} f(x)=q_{1} \forall x \in \mathcal{D}$.

So a map between manifolds is an immersion if it has the same rank as the domain. Obviously the rank is independent of the local chart used.

When an immersion is 'well-behaved' it is called an embedding. For well-behaved we mean that it has to be an isomorphism onto its image with respect to the topology induced from the corresponding $\mathbb{R}^{q_{1}}$ by the local chart used [Spivak 1979].

Definition 8 The $C^{\infty}$ map $f: M_{1} \rightarrow M_{2}$ is an embedding if it is an immersion and it is an homomorphism onto its image.

Moreover, we have the following definition:

Definition 9 Suppose $M_{1} \subset M_{2} . M_{1}$ is a submanifold of $M_{2}$ if the identity map id $: M_{1} \rightarrow M_{2}$ is embedding. 


\section{Kinematic model for the general n-trailer}

Suppose we have a generalized n-trailer system with $m(m \leq n)$ of the trailers not directly attached at the center of the previous axle but at a positive distance $M_{i}$ from this point. Assume that each body is composed of one single axle, this being equivalent to the case where 2-axis bodies are present, modulo a state feedback (see [Tilbury et al. 1995]). The

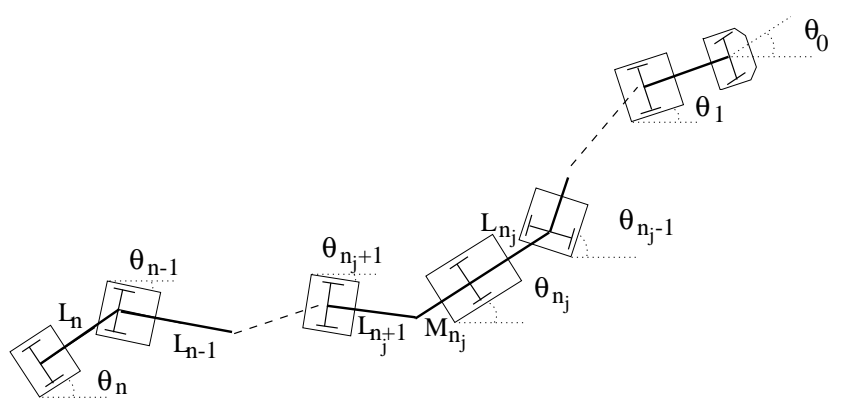

Figure 1: The general n-trailer system.

assumption of rolling without slipping of the wheels can be formulated in terms of nonholonomic kinematic constraints deciding the instantaneous direction of the velocity vector of each axle. Let $\theta_{i}$ be the orientation angle of the $i-t h$ axle and $v_{i}$ the translational velocity of the midpoint of the $i$-th axle, $i \in\{0,1, \ldots, n\}$. If $x_{i}$ and $y_{i}$ are the corresponding cartesian coordinates, then the one-forms can be expressed as:

$$
\alpha^{i}=d x_{i} \sin \theta_{i}-d y_{i} \cos \theta_{i}=0
$$

If the $L_{i}$ is the distance between the $i$-th axle and the hitching point of the same trailer and $M_{i}$ is the distance between the $i$-th axle and the kingpin hitching point of the following trailer, we can use the (holonomic) relations between two consecutive nodes $i-t h$ and $i+1-t h$ (see Fig. 1)

$$
\left\{\begin{array}{l}
x_{i+1}=x_{i}-L_{i+1} \cos \theta_{i+1}-M_{i} \cos \theta_{i} \\
y_{i+1}=y_{i}-L_{i+1} \sin \theta_{i+1}-M_{i} \sin \theta_{i}
\end{array}\right.
$$

and the one-forms $(6)$ to obtain a recursive equation for the orientation angle $\theta_{i+1}$ as a function of $\theta_{i}$ and $v_{i}, i \in\{1, \ldots, n-1\}$ :

$$
\dot{\theta}_{i+1}=\frac{v_{i} \sin \left(\theta_{i}-\theta_{i+1}\right)}{L_{i+1}}-\frac{M_{i} \cos \left(\theta_{i}-\theta_{i+1}\right) \dot{\theta}_{i}}{L_{i+1}}
$$

Also the calculation of the velocity of the axle $i+1$ is slightly more complicated than in the standard n-trailer problem:

$$
v_{i+1}=v_{i} \cos \left(\theta_{i}-\theta_{i+1}\right)+M_{i} \sin \left(\theta_{i}-\theta_{i+1}\right) \dot{\theta}_{i}
$$

This accounts for the intuitive phenomenon that, in presence of off-hitching, a trailer can have a (small) positive velocity and the following trailer a negative one, when the angle between 
the two trailers $\theta_{i}-\theta_{i+1}$ is changing with high rate. Obviously, both eq. (8) and eq. (9) reduce to the well-known equations for the standard n-trailer problem when no off-hitching is present, namely when $M_{i}=0$.

The n-trailer system has two physical inputs, corresponding to translational and steering

actions of the car pulling the trailers. Calling $\beta_{1} \triangleq \theta_{0}-\theta_{1}$, at the kinematic level we can consider these two inputs to be the steering speed $\omega_{0}=\dot{\beta}_{1}=\dot{\theta}_{0}-\dot{\theta}_{1}$ and the translational speed $v_{0}$ of the driving cart. Alternatively as steering input we can consider the following:

$$
\omega_{0}=\dot{\theta}_{0}=\frac{v_{0} \sin \beta_{1}+L_{1} \dot{\beta}_{1}}{L_{1}+M_{0} \cos \beta_{1}}
$$

To complete the state space model of the general n-trailer system, we need the cartesian coordinates of one of the middle points of the axles: for the purposes of proving controllability it is convenient to choose $\left(x_{0}, y_{0}\right)$ of the driving cart,

$$
\begin{aligned}
& \dot{x}_{0}=v_{0} \cos \theta_{0} \\
& \dot{y}_{0}=v_{0} \sin \theta_{0}
\end{aligned}
$$

whereas it has been shown in [Sørdalen 1993a] for the standard n-trailer problem, that choosing the ones of the last trailer is particularly significant when the task is to transform the system into chained form, because it is connected to differential flatness [Fliess et al. 1995].

$$
\begin{aligned}
& \dot{x}_{n}=v_{n} \cos \theta_{n} \\
& \dot{y}_{n}=v_{n} \sin \theta_{n}
\end{aligned}
$$

In fact, the coordinates $x_{n}$ and $y_{n}$ correspond to the so-called flat outputs for the standard n-trailer. In both cases, an ad hoc selection of the cartesian coordinates greatly simplifies the calculations.

The relation between $v_{0}$ and $v_{n}$ will be derived in Section 9.

\section{The virtual steering wheels}

The basic idea is that the $n$-trailer system with $m$ off-hitching joints can be converted into an $n+m$-trailer system with $m+1$ steering axles, adding a steerable wheel at each of the aforementioned joints not directly hitched on the preceding axle. These $m$ virtual steering wheels are passive, in the sense that their steering angles are (uniquely) determined by the configuration and by the dynamic equations of the system. This is equivalent to say that their inputs are obtained by means of feedback from the configuration of the system and only the driving unit has exogenous input.

First we prove that a passive steering wheel is indeed admissible by the system and then, in Section 6, we show that these virtual steering wheels provide physical insight into the extra singularities of the system due to the kingpin hitching.

Proposition 1 Consider the D-trailer off-hitching connection between the trailers $i$ and $i+1$. This subsystem is equivalent to a standard 3-trailer system with a steering wheel in the middle 


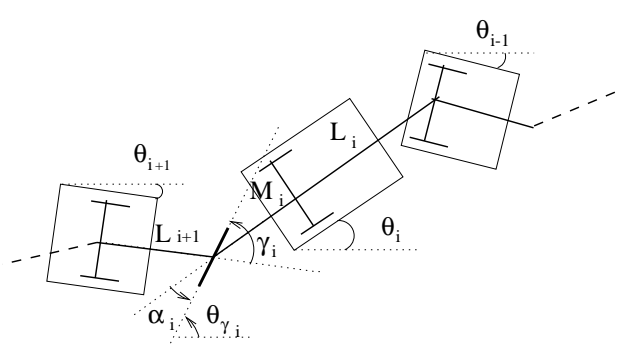

Figure 2: The virtual steering wheel.

(Fig. 2). If $\theta_{\gamma_{i}}$ is the orientation angle of the steering wheel and its steering angle is defined as $\gamma_{i} \triangleq \theta_{\gamma_{i}}-\theta_{i+1}$, then it must be:

$$
\gamma_{i}=\theta_{i}-\theta_{i+1}+\arctan \left(-\frac{M_{i}}{v_{i}} \dot{\theta}_{i}\right)
$$

Proof. Consider the sketch in Fig. 2. If such a virtual wheel exist, then, due to the rigidity of the connection between $M_{i}$ and $L_{i}$, the dynamic equation for the $i$-th orientation angle $\theta_{i}$, obtained in general from eq. (8), must also be expressible as a function of the angle $\alpha_{i}$ (representing the steering angle of the virtual wheel with respect to the preceding trailer):

$$
\dot{\theta}_{i}=-\frac{v_{i}}{M_{i}} \tan \alpha_{i}
$$

where the minus sign reflects the fact that the wheel is following and not preceding the axle. At the same time, the steering angle of the virtual wheel with respect to the following trailer $i+1, \gamma_{i}$, must be such that

$$
\dot{\theta}_{i+1}=\frac{v_{i+1}}{L_{i+1}} \tan \gamma_{i}
$$

Therefore, a physical solution exists (i.e. the wheel is admissible) iff

$$
\gamma_{i}=\theta_{i}-\theta_{i+1}+\alpha_{i}
$$

Substituting into eq. (8), we get:

$$
\frac{v_{i+1} \tan \gamma_{i}}{L_{i+1}}=\frac{v_{i+1} \tan \left(\theta_{i}-\theta_{i+1}\right)}{L_{i+1}}+\frac{M_{i} v_{i} \tan \alpha_{i}}{M_{i} L_{i+1} \cos \left(\theta_{i}-\theta_{i+1}\right)}
$$

using eq. (9):

$$
\begin{array}{r}
{\left[v_{i} \cos \left(\theta_{i}-\theta_{i+1}\right)+M_{i} \sin \left(\theta_{i}-\theta_{i+1}\right)\left(-\frac{v_{i}}{M_{i}} \tan \alpha_{i}\right)\right]} \\
\cdot\left(\tan \gamma_{i}-\tan \left(\theta_{i}-\theta_{i+1}\right)\right)=\frac{v_{i} \tan \alpha_{i}}{\cos \left(\theta_{i}-\theta_{i+1}\right)}
\end{array}
$$

i.e.

$$
\tan \gamma_{i}=\frac{\tan \left(\theta_{i}-\theta_{i+1}\right)+\tan \alpha_{i}}{1-\tan \left(\theta_{i}-\theta_{i+1}\right) \tan \alpha_{i}}=\tan \left(\theta_{i}-\theta_{i+1}+\alpha_{i}\right)
$$




\section{Comparison between standard and general $n$-trailer systems}

In what follows it is convenient to use the following notation: we call $n_{1}, \ldots, n_{m}, n_{j}<n_{j+1}$, $n_{m}<n$ the indices of the axles having nonnull off-hitching $\left(M_{n_{j}} \neq 0\right)$. We can group together the axles between two consecutive steering wheels: $\left\{0,1, \ldots, n_{1}\right\}, \ldots,\left\{n_{j-1}+1, \ldots, n_{j}-1, n_{j}\right\}$, $\ldots,\left\{n_{m}+1, \ldots, n-1, n\right\}$. Each of the groups of axles, together with the steering wheel in front of it, will constitute a steering train. In case of two consecutive axles having offhitching, then a steering train is reduced to a single axle. We call this a degenerate steering train. With this notation we can rewrite the dynamic equations of the orientation angles of the general $n$-trailer problem as:

$$
\begin{aligned}
\dot{\theta}_{n_{j}+1}= & \frac{v_{n_{j}+1} \tan \left(\theta_{n_{j}}-\theta_{n_{j}+1}\right)}{L_{n_{j}+1}}-\frac{M_{n_{j}} \dot{\theta}_{n_{j}}}{L_{n_{j}+1} \cos \left(\theta_{n_{j}}-\theta_{n_{j}+1}\right)} \\
v_{n_{j}+1}= & v_{n_{j}} \cos \left(\theta_{n_{j}}-\theta_{n_{j}+1}\right)+M_{n_{j}} \sin \left(\theta_{n_{j}}-\theta_{n_{j}+1}\right) \dot{\theta}_{n_{j}} \\
& j \in\{1, \ldots, m\} \\
\dot{\theta}_{n_{j}-i}= & \frac{v_{n_{j}-i} \tan \left(\theta_{n_{j}-i-1}-\theta_{n_{j}-i}\right)}{L_{n_{j}-i}} \\
v_{n_{j}-i}= & v_{n_{j}-i-1} \cos \left(\theta_{n_{j}-i-1}-\theta_{n_{j}-i}\right)
\end{aligned}
$$

with $j \in\{1, \ldots, m+1\}, i \in\left\{0,1, \ldots, n_{j}-n_{j-1}-2\right\}$ and $n_{m+1}=n$.

We assume, in what follows, that the steering trains are not degenerate.

In order to highlight the similarities of the dynamic eq. (16)-(19) with those of the standard $n$-trailer it is convenient to rewrite them in terms of the relative orientation angles $\beta_{i} \triangleq \theta_{i-1}-\theta_{i}, i \in\{1, \ldots, n\}$, expressing all $v_{i}$ as functions of $v_{0}$ and using the cartesian coordinates $\left(x_{0}, y_{0}\right)$. The system becomes:

$$
\begin{aligned}
& \dot{x}_{0}=v_{0} \cos \theta_{0} \\
& \dot{y}_{0}=v_{0} \sin \theta_{0} \\
& \dot{\theta}_{0}=\omega_{0} \\
& \dot{\beta}_{1}=-\frac{v_{0} \sin \beta_{1}}{L_{1}}+\omega_{0} \\
& \dot{\beta}_{n_{j}+1}=v_{0}\left(\prod_{k=1}^{n_{j}} \cos \beta_{k}\right) \prod_{k=1}^{j-1}\left(1+\frac{M_{n_{k}}}{L_{n_{k}}} \tan \beta_{n_{k}} \tan \beta_{n_{k}+1}\right) \\
& \cdot\left(\frac{\tan \beta_{n_{j}}}{L_{n_{j}}}-\frac{\sin \beta_{n_{j}+1}}{L_{n_{j}+1}}+\frac{M_{n_{j}}}{L_{n_{j}} L_{n_{j}+1}} \tan \beta_{n_{j}} \cos \beta_{n_{j}+1}\right) \\
& \dot{\beta}_{n_{j}-i}=v_{0}\left(\prod_{k=1}^{n_{j}-i-1} \cos \beta_{k}\right) \prod_{k=1}^{j-1}\left(1+\frac{M_{n_{k}}}{L_{n_{k}}} \tan \beta_{n_{k}} \tan \beta_{n_{k}+1}\right) \\
& \cdot\left(\frac{\tan \beta_{n_{j}-i-1}}{L_{n_{j}-i-1}}-\frac{\sin \beta_{n_{j}-i}}{L_{n_{j}-i}}\right)
\end{aligned}
$$


$j \in\{1, \ldots, m\}, i \in\left\{0,1, \ldots, n_{j}-n_{j-1}-2\right\}$.

Notice that equations (20)-(25) are everywhere well defined.

Clearly, each of the components due to the kingpin hitching enters linearly into the system as an extra term added to the basic dynamic equations of the standard $n$-trailer, which are reobtained choosing $M_{n_{j}}=0$ :

$$
\begin{aligned}
\dot{x}_{0} & =v_{0} \cos \theta_{0} \\
\dot{y}_{0} & =v_{0} \sin \theta_{0} \\
\dot{\theta}_{0} & =\omega_{0} \\
\dot{\beta}_{1} & =-\frac{v_{0} \sin \beta_{1}}{L_{1}}+\omega_{0} \\
\dot{\beta}_{i+1} & =v_{0}\left(\prod_{k=1}^{i} \cos \beta_{k}\right)\left(\frac{\tan \beta_{i}}{L_{i}}-\frac{\sin \beta_{i+1}}{L_{i+1}}\right)
\end{aligned}
$$

$i \in\{1, \ldots, n-1\}$.

In the rest of the paper we will pass indifferently form the coordinate system in the absolute angles $\theta_{i}, i=1, \ldots, n$, to the other one in the relative orientation angles $\beta_{i}, i=$ $1, \ldots, n$, according to convenience.

The Pfaffian system associated with (16)-(19) is simply the collection of one-forms (6) plus the equations between pairs of cartesian coordinates of two adjacent nodes (7). So the irregularities enter only into the holonomic relations linking two consecutive one-forms via a kingpin hitch and propagate to their exterior derivatives:

$$
\begin{aligned}
d x_{n_{j}} & =d x_{n_{j}+1}-L_{n_{j}+1} \sin \theta_{n_{j}+1} d \theta_{n_{j}+1}-M_{n_{j}} \sin \theta_{n_{j}} d \theta_{n_{j}} \\
d y_{n_{j}} & =d y_{n_{j}+1}+L_{n_{j}+1} \cos \theta_{n_{j}+1} d \theta_{n_{j}+1}+M_{n_{j}} \cos \theta_{n_{j}} d \theta_{n_{j}}
\end{aligned}
$$

$j \in\{1, \ldots, m\}$. When off-hitching is missing instead we have:

$$
\begin{aligned}
d x_{n_{j}-i-1} & =d x_{n_{j}-i}-L_{n_{j}-i} \sin \theta_{n_{j}-i} d \theta_{n_{j}-i} \\
d y_{n_{j}-i-1} & =d y_{n_{j}-i}+L_{n_{j}-i} \cos \theta_{n_{j}-i} d \theta_{n_{j}-i}
\end{aligned}
$$

$j \in\{1, \ldots, m+1\}, i \in\left\{0,1, \ldots, n_{j}-n_{j-1}-2\right\}$. In order to recover the original configuration space dimension, we have to substitute into (6) the cartesian coordinates as functions of one single axle. In a proper domain of definition (see below) we can rewrite the one-forms (6) in terms of congruences:

$$
d y_{i} \equiv \tan \theta_{i} d x_{i} \bmod \alpha^{i} \quad i \in\{0,1, \ldots, n\}
$$

meaning with this expression that the congruence is satisfied up to an element of the ideal generated by $\alpha^{i}$ i.e. $d y_{i}=\tan \theta_{i} d x_{i}+\gamma \wedge \alpha^{i}$ for some $\gamma \in \Omega(M)$ also satisfies it. In [Pappas et al. 1998] it is shown how to use the congruence to obtain a relation between the exterior derivatives of the cartesian coordinates of two adjacent standard trailers. This is basically what we need in order to eliminate the holonomic constraints on the Pfaffian system. With the same notation convention used above, In the standard case, see Fig. 3 (a), 


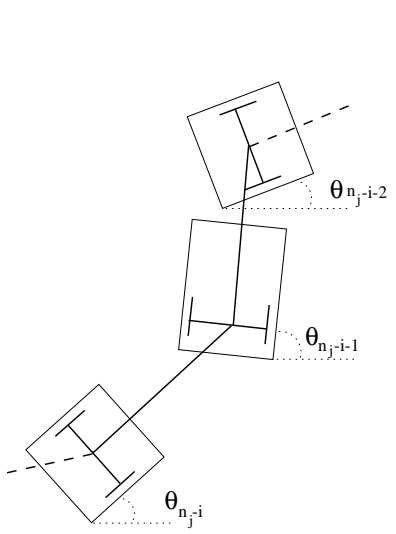

(a) standard hitching: $M_{n_{j}-i-2}=M_{n_{j}-i-1}=$ 0

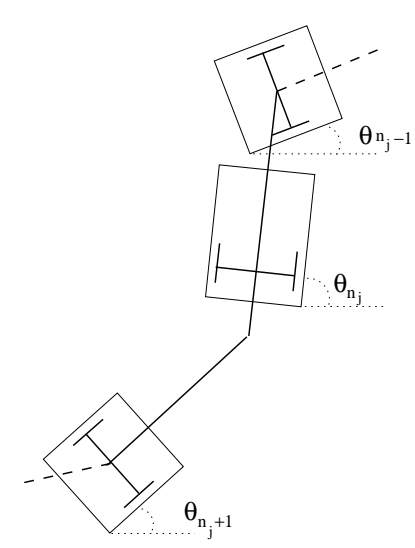

(b) off-axle hitching: $M_{n_{j}-1}=0, M_{n_{j}} \neq 0$

Figure 3: The two different kinds of hitching.

at the node $n_{j}-i$ the congruence between the exterior derivatives of the orientation angle $\theta_{n_{j}-i}$ and that of the corresponding cartesian coordinate $x_{n_{j}-i}$ is modulo the two one-forms $\alpha^{n_{j}-i-1}$ and $\alpha^{n_{j}-i}$. We get in the case $M_{n_{j}-i-2}=M_{n_{j}-i-1}=0$ :

$$
d \theta_{n_{j}-i} \equiv \frac{\tan \left(\theta_{n_{j}-i-1}-\theta_{n_{j}-i}\right)}{L_{n_{j}-i} \cos \theta_{n_{j}-i}} d x_{n_{j}-i} \quad \bmod \alpha^{n_{j}-i-1}, \alpha^{n_{j}-i}
$$

which gives the recursive relation

$$
d x_{n_{j}-i-1} \equiv\left(1-\tan \theta_{n_{j}-i} \tan \left(\theta_{n_{j}-i-1}-\theta_{n_{j}-i}\right)\right) d x_{n_{j}-i} \bmod \alpha^{n_{j}-i-1}, \alpha^{n_{j}-i}
$$

When, instead, a joint with kingpin hitch is involved, Fig. 3 (b), the formulae for the congruences gets more complicated:

$$
d \theta_{n_{j}+1} \equiv \frac{\tan \left(\theta_{n_{j}}-\theta_{n_{j}+1}\right)-\frac{M_{n_{j}}}{L_{n_{j}}} \tan \left(\theta_{n_{j}-1}-\theta_{n_{j}}\right)}{L_{n_{j}+1} \cos \theta_{n_{j}+1}\left(1+\frac{M_{n_{j}}}{L_{n_{j}}} \tan \left(\theta_{n_{j}-1}-\theta_{n_{j}}\right) \tan \left(\theta_{n_{j}}-\theta_{n_{j}+1}\right)\right)} d x_{n_{j}+1} \quad \bmod \alpha^{n_{j}-1}, \alpha^{n_{j}}, \alpha^{n_{j}+1}
$$

which says that the congruence following a kingpin hitch involves three (instead of two) consecutive one-forms. This also appears in the recursive calculation of the $d x_{i}$ :

$$
\begin{aligned}
d x_{n_{j}} \equiv & \frac{1-\tan \theta_{n_{j}+1} \tan \left(\theta_{n_{j}}-\theta_{n_{j}+1}\right)+\frac{M_{n_{j}}}{L_{n_{j}}} \tan \left(\theta_{n_{j}-1}-\theta_{n_{j}}\right)\left(\tan \left(\theta_{n_{j}}-\theta_{n_{j}+1}\right)-\tan \theta_{n_{j}+1}\right)}{\left(1+\frac{M_{n_{j}}}{L_{n_{j}}} \tan \theta_{n_{j}} \tan \left(\theta_{n_{j}}-\theta_{n_{j}+1}\right)\right)\left(1+\frac{M_{n_{j}}}{L_{n_{j}}} \tan \left(\theta_{n_{j}-1}-\theta_{n_{j}}\right) \tan \left(\theta_{n_{j}}-\theta_{n_{j}+1}\right)\right)} d x_{n_{j}+1} \\
& \bmod \alpha^{n_{j}-1}, \alpha^{n_{j}}, \alpha^{n_{j}+1}
\end{aligned}
$$

What these complicated formulae tell us, as well as their vector field counterpart (24)-(25), is the (intuitively clear) fact that the presence of off-hitching implies that a motion with 
nonnull steering propagates more 'in depth' in the chain of trailers than in the standard configuration, i.e. the change of the orientation angle in the trailer that follows the off-axle hitch is influenced not only by the orientation angle of the trailer in front of it as in the standard case, but also by the one of second trailer ahead. When the system has degenerate steering trains (i.e. it has two or more consecutive axles with kingpin hitching), the number of one-forms involved into a relation like (34) is higher than three. We will see in Section 7 that this argument, in particular its formulation on the dual system, can help in understanding the local controllability property of the general $n$-trailer.

Putting together all equations for adjacent axles we get that the following proposition still holds true also for the general $n$-trailer.

Proposition 2 ( [Pappas et al. 1998], Lemma 42 ) The exterior derivatives of any of the $x$ variables are congruent modulo the Pfaffian system I:

$$
d x_{i} \equiv f_{x_{i, j}} d x_{j} \bmod I \text {. }
$$

All the above reasoning has value only locally, in a (usually large enough) interval containing the origin. The set of points in which the model formulation is undefined is larger in the general $n$-trailer that in the standard $n$-trailer. Its physical meaning is explained in next Section.

\section{Singular locus}

The index at which the filtration stops at a point $x_{0}$ is called the degree of nonholonomy of the system at $x_{0}$. When $x_{0}$ satisfies the Lie algebra rank condition then the system is said maximally nonholonomic at $x_{0}$. Singular points for the standard $n$-trailer system have the physical meaning of orthogonal angles between consecutive trailers and they lead to a different degree of nonholonomy, see [Jean 1996] for a complete discussion.

For the general $n$-trailer, proposition 1 affirms that a virtual steering wheel can be placed on the kingpin hitching nodes and gives a value for its steering angle. Furthermore, from eq. (15) we see that the tangent of the virtual steering angle enters into the dynamic equation of the heading of the following trailer. Therefore one can ask whether the 'virtual' singularity introduced by $\tan \gamma_{j}$ must be considered in the analysis of the controllability of the model or less. The corresponding singular points can be rewritten using the formula:

$$
\alpha_{j}=\arctan \left(-\frac{M_{n_{j}}}{v_{n_{j}}} \dot{\theta}_{n_{j}}\right)=\gamma_{j}-\beta_{n_{j}+1}
$$

as

$$
\dot{\theta}_{n_{j}}=-\frac{v_{n_{j}}}{M_{n_{j}}} \tan \left(\gamma_{j}-\beta_{n_{j}+1}\right)=\frac{v_{n_{j}}}{L_{n_{j}}} \tan \beta_{n_{j}}
$$

or, after some manipulations,

$$
\tan \gamma_{j}=\frac{\tan \beta_{n_{j}+1}-\frac{M_{n_{j}}}{L_{n_{j}}} \tan \beta_{n_{j}}}{1+\frac{M_{n_{j}}}{L_{n_{j}}} \tan \beta_{n_{j}+1} \tan \beta_{n_{j}}}
$$


The singular point

$$
\gamma_{j} \rightarrow \frac{\pi}{2} \bmod \pi
$$

is then equivalent to

$$
1+\frac{M_{n_{j}}}{L_{n_{j}}} \tan \beta_{n_{j}+1} \tan \beta_{n_{j}} \rightarrow 0 .
$$

With eq. (36), the question posed above can now easily be answered looking at eq. (24)(25): the 'virtual' singularities are indeed singular points of the control Lie algebra since $\left(1+\frac{M_{n_{j}}}{L_{n_{j}}} \tan \beta_{n_{j}+1} \tan \beta_{n_{j}}\right)=0$ implies that the vector field associated with the input $v_{0}$ has the last $n-n_{j}-1$ components that are null. From eq. (24)-(25), also the $\cos \beta_{k}$ have the same effect of annulling the last $n-k$ terms of the input vector field associated to $v_{0}$. The consequence is that the Lie bracket cannot generate a full rank distribution with the same growth vector as in the nearby points. Similarly to the standard case, the proof should pass through the computation of the filtration (4). However, an informal argument can be given by looking at the two vector fields in (23)-(25): the vector field associated with the input $\omega_{0}$ has only the first component different from zero and it is a constant, while the vector field relative to $v_{0}$ has a triangular structure. Therefore, whenever there are null components due to $\cos \beta_{k}$ or to $\left(1+\frac{M_{n_{j}}}{L_{n_{j}}} \tan \beta_{n_{j}+1} \tan \beta_{n_{j}}\right)$ all the remaining Lie brackets will be zero. These last extra singularities, which are not present in the standard n-trailer system, have then the 'nice' physical interpretation of one of the virtual wheels being orthogonal to the velocity vector of the following trailer.

To summarize: in the standard $n$-trailer we have that the singular locus is given by (see [Jean 1996])

$$
\mathcal{S}_{S}=\left\{\beta_{i}=\frac{\pi}{2} \bmod \pi\right\} ; i \in\{1, \ldots, n\}
$$

whereas, in the general $n$-trailer, it is larger:

$$
\mathcal{S}_{G}=\left\{\beta_{i}=\frac{\pi}{2} \bmod \pi\right\} \bigcup\left\{\gamma_{j}=\frac{\pi}{2} \bmod \pi\right\}
$$

$i \in\{1, \ldots, n\}, j \in\{1, \ldots, m\}$.

Like $\mathcal{S}_{S}, \mathcal{S}_{G}$ is a set of measure zero in the configuration space of the system.

For the standard $n$-trailer there exist techniques that allow to calculate the exact value of the degree of nonholonomy in the singular locus [Jean 1996]. Also for the general $n$-trailer it should be possible to obtain a similar procedure, although the basis of vector fields at the singular points might result even more complicated that the one proposed in [Jean 1996].

Singular locus and domain of definition The singularity analysis mentioned above is not invariant to the selection of coordinates for the system. In particular, it can be noticed that the singular locus is related to the domain of definition of the kinematic model when we change the velocity input by means of a (locally) invertible transformation.

In our case, if we assume to take as longitudinal input $v_{n}$ instead of $v_{0}$, then the velocities of all the other trailers can be calculated consequently. For a generic axle $n-i$-th on the 


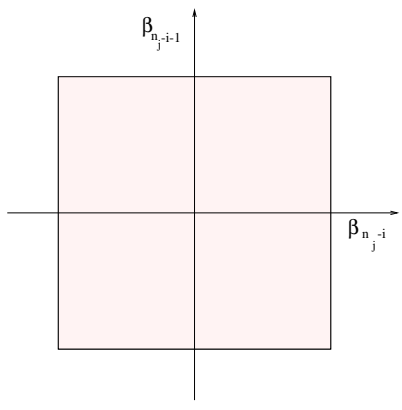

(a) standard $n$-trailer

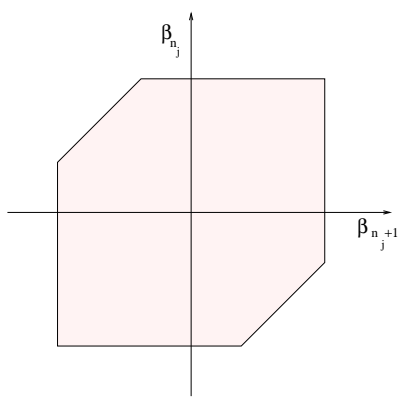

(b) general $n$-trailer

Figure 4: Singular locus and connected component containing the origin in which the system is regular with respect to the filtration (grey areas) of the standard and general $n$-trailers.

last steering train we get the usual formula:

$$
v_{n-i}=\frac{v_{n}}{\prod_{l=n-i}^{n-1} \cos \left(\theta_{l}-\theta_{l+1}\right)}
$$

whereas for the last trailer of the second-to-last steering train we have, if the $m$-th steering train is not degenerate,

$$
v_{n_{m}}=\frac{v_{n}}{\left(1+\frac{M_{n_{m}}}{L_{n_{m}}} \tan \left(\theta_{n_{m}-1}-\theta_{n_{m}}\right) \tan \left(\theta_{n_{m}}-\theta_{n_{m}+1}\right)\right) \prod_{l=n_{m}}^{n-1} \cos \left(\theta_{l}-\theta_{l+1}\right)}
$$

The case of degenerate steering trains leads to a different (more complicated) expression, according to the number of consecutive degenerate trains. For a generic axle $n_{j}-i(i+1$-th rearmost trailer of the $j$-th steering train) we have:

$$
v_{n_{j}-i}=\frac{v_{n}}{\prod_{k=j}^{m}\left(1+\frac{M_{n_{k}}}{L_{n_{k}}} \tan \left(\theta_{n_{k}-1}-\theta_{n_{k}}\right) \tan \left(\theta_{n_{k}}-\theta_{n_{k}+1}\right)\right) \prod_{l=n_{j}-i}^{n-1} \cos \left(\theta_{l}-\theta_{l+1}\right)}
$$

and for the pulling cart:

$$
v_{0}=\frac{v_{n}}{\prod_{k=1}^{m}\left(1+\frac{M_{n_{k}}}{L_{n_{k}}} \tan \left(\theta_{n_{k}-1}-\theta_{n_{k}}\right) \tan \left(\theta_{n_{k}}-\theta_{n_{k}+1}\right)\right) \prod_{l=0}^{n-1} \cos \left(\theta_{l}-\theta_{l+1}\right)}-M_{0} \tan \left(\theta_{0}-\theta_{1}\right) \omega_{0}
$$

For sake of simplicity, we assume that $v_{n}$ can be expressed as a homogeneous linear function of of $v_{0}$. This is equivalent to say that we do not want off-hitching on the leading car $\left(M_{0}=0\right)$.

So $\mathcal{S}_{G}$ is now the set of points in which the equations (20)-(25) of the system, rewritten as functions of $v_{n}$ are not defined. We would like to remark that this observation is true also for the standard $n$-trailer, as can be easily deducted rewriting the equations (26)-(30) as functions of $v_{n}$ (with all $M_{i}=0$ ). What is interesting to notice is that in both cases the region 
inside the singular locus, depicted in Fig. 4, corresponds to the domain of definition (again connected component containing the origin of the domain of definition) of the formulation that takes $v_{n}$ as velocity input.

\section{Controllability of the general $n$-trailer}

One of the major features of the nonholonomic wheeled systems is that they constitute a class of systems for which the analysis on the Jacobian linearization often fails to give the right result, which can be instead obtained using nonlinear techniques. This is the case for the controllability property: the standard $n$-trailer is not linearly controllable (it is a driftfree system and it is underactuated) but it was shown to be controllable by Laumond (see for example [Laumond 1990]) using the methods described in Section 2. For this system, the main problem is to verify the rank condition for a generic value of $n$, which means the need of having an iterative procedure like the one proposed by Laumond [Laumond 1993b], and to cope with singularities. Out of the singularity locus it is possible to state a similar local property for the general $n$-trailer configuration.

Theorem 1 The general n-trailer system (20)-(25) is locally controllable out of the singular locus $\mathcal{S}_{G}$.

Proof. In our point of view it is interesting to see both the ways of checking local controllability given by the two dual versions of the Chow theorem reported in Section 2, via the two practical iterative tests illustrated in the same Section. Therefore we show below both proofs in order to be able to make a comparison.

Version 1: vector field formulation.

Since the rigorous mathematical formulation requires a lot of bookkeeping without adding anything significant to the discussion, we provide only a somewhat informal argument.

Basically the proof is a direct consequence of the bilinearity of the Lie bracket operation:

$$
\left[X_{1}+X_{2}, Y\right]=\left[X_{1}, Y\right]+\left[X_{2}, Y\right]
$$

In fact, from eq. (20)-(25) we have that the vector fields that form the distribution for the general $n$-trailer $\Delta_{G}=\operatorname{span}\left\{g_{1_{G}}, g_{2_{G}}\right\}$ can be written as the sum of the corresponding $g_{1_{S}}, g_{2_{S}}$ of the standard $n$-trailer plus an extra component on the vector field associated with the tangential speed input $v_{0}$ :

$$
\begin{aligned}
& g_{1_{G}}=g_{1_{S}}+g_{1_{H}} \\
& g_{2_{G}}=g_{2_{S}}
\end{aligned}
$$

The first $n_{1}+3$ components of $g_{1_{H}}$ are equal to 0 . If we call:

$$
p_{j} \triangleq \frac{M_{n_{j}}}{L_{n_{j}}} \tan \beta_{n_{j}} \tan \beta_{n_{j}+1} \quad j \in\{1, \ldots, m\}
$$


then the generic $n_{j}+i+3$ element of the vector $g_{1_{H}}$ is:

$g_{1_{H}}\left[n_{j}-i+3\right]=\left(\prod_{k=1}^{n_{j}-i-1} \cos \beta_{k}\right) \sum_{k=1}^{j-1}\left(\sum_{l(k) \in C(k, j-1)}\left(p_{l(1)} \cdot \ldots \cdot p_{l(k)}\right)\right)\left(\frac{\tan \beta_{n_{j}-i-1}}{L_{n_{j}-i-1}}-\frac{\sin \beta_{n_{j}-i}}{L_{n_{j}-i}}\right)$

where $C(k, j-1)$ is the set of possible combinations of $k$ numbers in the first $j-1$ integers. The component $n_{j}+4$, representing the equation of the axle immediately after the kingpin hitching, has a slightly different expression:

$$
\begin{gathered}
g_{1_{H}}\left[n_{j}+4\right]=\left(\prod_{k=1}^{n_{j}} \cos \beta_{k}\right) \frac{M_{n_{j}} \tan \beta_{n_{j}} \cos \beta_{n_{j}+1}}{L_{n_{j}} L_{n_{j}+1}} \\
+\left(\prod_{k=1}^{n_{j}} \cos \beta_{k}\right) \sum_{k=1}^{j-1}\left(\sum_{l(k) \in C(k)}\left(p_{l(1)} \ldots \cdot p_{l(k)}\right)\right)\left(\frac{\tan \beta_{n_{j}}}{L_{n_{j}}}-\frac{\sin \beta_{n_{j}+1}}{L_{n_{j}+1}}+\frac{M_{n_{j}} \tan \beta_{n_{j}} \cos \beta_{n_{j}+1}}{L_{n_{j}} L_{n_{j}+1}}\right)
\end{gathered}
$$

Now, we can use the result of Laumond for the standard $n$-trailer. In [Laumond 1993b] a family of vector fields is proposed that generates the control Lie algebra for the standard $n$-trailer. The family is composed of $n+3$ vector fields for any value of $n$. Out of the singular locus $\mathcal{S}_{G}$, the result still holds also for our $\Delta_{G}$ in the sense that the vector fields of the family remain independent as in the standard case. The rank condition being satisfied, the system is locally controllable in any regular point. In the case of [Laumond 1993b], the controllability property was verified everywhere. Here, due to the more complicated formulae, it is not easy to understand how to proceed at the singular points.

Version 2: derivative flag analysis.

We saw that the system is not controllable if the ideal generated by the Pfaffian system or by a nonempty subset of it satisfies the Frobenius theorem. So we use the derivative flag to calculate the largest integrable subsystem of the codistribution $I$. In order to compute the derivative flag $I^{(j)}$, we need to calculate the exterior derivative of the constraints and to determine the set of one-forms that each of those derivatives requires in order to be congruent to 0 . When calculating the exterior derivative of one of the constraints $\alpha^{i}, i \in\{0,1, \ldots, n\}$, we obtain:

$$
d \alpha^{i} \equiv \frac{d \theta_{i} \wedge d x_{i}}{\cos \theta_{i}} \quad \bmod \alpha^{i}
$$

where we have used the fact that the two-form $d^{2} x_{i}$ is equal to 0 by definition of exterior derivative. If we now substitute $d \theta_{i}$ with the corresponding expressions (32) or (33) according to whether the axle is connected without or with kingpin hitch to the trailer in front of it, then we obtain congruences to 0 in both cases, but modulo a different set of constraints:

$$
d \alpha^{n_{j}-i} \equiv 0 \bmod \alpha^{n_{j}-i}, \alpha^{n_{j}-i-1}, j \in\{1, \ldots, m+1\}, i \in\left\{0,1, \ldots, n_{j}-n_{j-1}-2\right\}
$$

for the standard connection, and

$$
d \alpha^{n_{j}+1} \equiv 0 \bmod \alpha^{n_{j}-1}, \alpha^{n_{j}}, \alpha^{n_{j}+1}, j \in\{1, \ldots, m\}
$$

for the off-axle hitches. So in the first case the exterior derivative is a linear combination of two one-forms (via coefficient that are forms in $\Omega(M)$ and wedge product) whereas in the second case the linear combination has to be done over three consecutive one-forms. This 
spoils the regularity of the derivative flag in the sense that any time you hit a kingpin hitch the next derived Pfaffian system will loose two elements instead of one as in the standard case. The derivative flag goes as follows:

$$
\begin{aligned}
& I^{(0)}=\left\{\begin{array}{llllllllll}
\alpha^{0} & \alpha^{1} & \alpha^{2} & \ldots & \alpha^{n_{1}-1} & \alpha^{n_{1}} & \alpha^{n_{1}+1} & \alpha^{n_{1}+2} & \ldots & \alpha^{n}
\end{array}\right\} \\
& I^{(1)}=\left\{\begin{array}{lllllllll}
\alpha^{1} & \alpha^{2} & \ldots & \alpha^{n_{1}-1} & \alpha^{n_{1}} & \alpha^{n_{1}+1} & \alpha^{n_{1}+2} & \ldots & \alpha^{n}
\end{array}\right\} \\
& I^{(2)}=\left\{\begin{array}{llllllll}
\alpha^{2} & \ldots & \alpha^{n_{1}-1} & \alpha^{n_{1}} & \alpha^{n_{1}+1} & \alpha^{n_{1}+2} & \ldots & \alpha^{n}
\end{array}\right\} \\
& I^{\left(n_{1}-1\right)}=\left\{\begin{array}{llllll}
\alpha^{n_{1}-1} & \alpha^{n_{1}} & \alpha^{n_{1}+1} & \alpha^{n_{1}+2} & \ldots & \alpha^{n}
\end{array}\right\} \\
& I^{\left(n_{1}\right)}=\left\{\begin{array}{lllll}
\alpha^{n_{1}} & \alpha^{n_{1}+1} & \alpha^{n_{1}+2} & \ldots & \alpha^{n}
\end{array}\right\} \\
& I^{\left(n_{1}+1\right)}=\left\{\quad \alpha^{n_{1}+2} \ldots \alpha^{n}\right\} \\
& I^{(n-m)}=\left\{\quad \alpha^{n}\right\}
\end{aligned}
$$

So the derivative flag ends (i.e. becomes empty) quicker than in the standard case (see [Pappas et al. 1998]). Therefore, by the Chow theorem for Pfaffian systems the system is locally controllable.

Obviously, the verification of controllability in a global sense requires more effort and a technique like the one proposed by [Jean 1996] should be exploited, in order to deal with singularity analysis.

\section{The general $n$-trailer as an embedding}

In Section 4, we showed that a passive steering wheel is indeed admissible by the system in any of the off-axle joints. With the $m$ virtual steering wheels, the system is 'similar' (in a sense to be defined) to a standard $m+n$-trailer system with $m+1$ steering inputs with the peculiarity that $m$ of the $m+1$ steering inputs are not controls because they are fixed by the feedback law (14). In fact, a fundamental property of a control is that it is a free parameter, independent of the configuration state. The $m$ virtual angles can be interpreted as feedback loops where the feedback law is not chosen by the user but uniquely determined by the geometry of the problem. If we open those $m$ loops, we get a true $n+m$-trailer system with $m+1$ steering control inputs. Such a system lives on a manifold $M_{M}$ of dimension $n+2 m+5$ characterized by $n+m+3$ states ( 2 cartesian coordinates, the usual $n+1$ orientation angles of the nonsteerable trailers plus other $m$ orientation angles for the steerable carts, called $\theta_{\gamma_{i}}$ in Fig. 2) and $m+2$ inputs (a longitudinal velocity and $m+1$ steering inputs), see [Tilbury et al. 1995] for the details. If we now close the $m$ loops for the extra steering wheels according to the state feedback (14), we reobtain the general $n$-trailer (20)-(25) that can be considered as evolving on a manifold $M_{G}$ of dimension $n+5(n+3$ states plus 2 inputs). So the virtual loops locally reduce the dimension of the manifold of $2 m$ as the steering feedback channels are used to 'annihilate' the states corresponding to the angles of the steerable virtual carts with the effect of rigidly relate the $\theta_{\gamma_{i}}$ to the preceding steering

angles $\theta_{n_{j}}\left(\theta_{\gamma_{i}}=\theta_{n_{j}}\right.$ after closing the loop). The following proposition states that applying 
the feedback law (14) to the multisteering $n$-trailer corresponds to embedding the general $n$-trailer into the multisteering structure.

Proposition 3 The general n-trailer (20)-(25) is an embedding of the multisteering $n$-trailer via the virtual feedback law (14).

Proof. As seen above, the general $n$-trailer is evolving on the manifold $M_{G}$ of dimension $n+5$ and the multisteering $n$-trailer on $M_{M}$ of dimension $n+5+2 m$. Around the origin we are using a minimal representation for both systems i.e. locally the two manifolds are diffeomorphic to euclidean spaces of the same dimension. Moreover, proposition 1 establish a locally well-defined map from $M_{M}$ to $M_{G}$ through the idea of virtual feedback loops. Therefore, by dimension counting this is an immersion map from $M_{G}$ to $M_{M}$. As the virtual feedback defined by (14) is smooth and bounded in the sense that it uniformly tends to zero when the state of the multisteering $n$-trailer tends to zero, then locally the immersion map does not introduce any strange phenomenon and it is a nice topological isomorphism onto its image and therefore an embedding.

The message here is the following: the application of feedback (14) to a multisteering $n$-trailer restrict the system to a submanifold of its original manifold $M_{M}$. This submanifold is nothing but $M_{G}$, the manifold on which the general $n$-trailer is living. As usual, everything that happens on a proper submanifold has 'measure zero' on the original manifold, but what is really important here is that it is compatible with the bigger structure.

It is important to remark that the virtual steering wheels introduced here are not dynamic prolongations of the system. In fact the general $n$-trailer and the multisteering $n$-trailer with the $m$ feedback loops considered here live in the same manifold so they have the same dimension. Indeed opening the feedback loops around the virtual steering wheels something else is obtained, namely the true multisteering $n$-trailer.

\section{Conversion into chained form}

The chained form for driftless nonlinear control systems is important because it corresponds to a particularly nice diffeomorphic representation of the original system with important regularity properties.

It has been shown in [Tilbury et al. 1995] that a standard multisteering $n+m$-trailer system can be put into a multi-input chained form. The transformation consists, in practice, in considering each subsystem of trailers between two consecutive steering wheels as a chain with the last trailer of the train as bottom of the chain. The solution proposed by [Tilbury et al. 1995] holds for $m+1$ generic exogenous steering inputs, so it will continue to hold also under state feedback for the $m$ new steering inputs.

Transforming a system into chained form means applying a static change of input and a state diffeomorphism in order for the original system to appear 'nicer' i.e. to have some extra properties that simplify dealing with problems like motion planning, trajectory tracking, stabilization, etc. If the multisteering $n$-trailer can be converted into multiinput chained form, what happens when we apply the virtual feedback (14)? Such a feedback is a smooth 
$\operatorname{map}$

$$
\begin{aligned}
\omega: \mathcal{D} & \rightarrow \mathbb{R}^{m} \\
x & \mapsto \omega(x)
\end{aligned}
$$

If we apply a diffeomorphism to the state $x$ :

$$
\begin{aligned}
\psi: \mathcal{D} & \rightarrow \mathcal{D} \\
x & \mapsto z=\psi(x)
\end{aligned}
$$

then the feedback can be rewritten in terms of the new basis using the chain rule as

$$
\begin{aligned}
\omega: \mathcal{D} & \rightarrow \mathbb{R}^{m} \\
\psi^{-1}(z) & \mapsto \omega\left(\psi^{-1}(z)\right)
\end{aligned}
$$

As $\psi(\cdot)$ is a diffeomorphism, all the properties of the virtual feedback are preserved, therefore also the embedding property and the identification of $M_{G}$ with a submanifold of $M_{M}$ also in the $z$ coordinate chart. So it makes sense to convert the multisteering $n$-trailer into chained form as in [Tilbury et al. 1995] and then apply the state feedback (14) transformed into the new basis as in (41).

Unlike [Tilbury et al. 1995], the resulting system does not need any dynamic prolongation, the reason being that the bottom of each steering train is independent from the trailers following behind.

As mentioned above, the transformation to chained form is greatly simplified when the coordinates of the last trailer are considered, instead of the pulling car. In particular, the velocity of the last trailer (rescaled by $\cos \theta_{n}$ ) results being the generator of the whole multiinput chain. The system can be split into $m+1$ steering trains. Our aim is to transform these $m+1$ subsystems into $m+12$-input chained forms, all with the same generator, only through differentiation. The generating input will be proportional to $v_{n}$. It is convenient to choose as generating input $v \triangleq v_{n} \cos \theta_{n}$ because this give immediately $\dot{x}_{n}=v$ so that we can choose the state of the short chain as $z_{g} \triangleq x_{n}$. The corresponding bottoms of the chains will be the other cartesian coordinate for the last chain and the $\theta_{n_{j}}, j \in\{1, \ldots, m\}$, i.e. the orientation angles of the trailers off-hitched, for the first $m$ chains. According to eq. (1), and to eq. (38), the dynamic equation for the orientation angle immediately following the $j$-th virtual wheel can be written as:

$$
\begin{aligned}
\dot{\theta}_{n_{j}+1}= & \frac{v_{n_{j}+1}}{L_{n_{j}+1}} \tan \gamma_{j} \\
= & \frac{v \tan \left(\theta_{n_{j}}-\theta_{n_{j}+1}+\arctan \left(-\frac{M_{n_{j}}}{L_{n_{j}}} \tan \left(\theta_{n_{j}-1}-\theta_{n_{j}}\right)\right)\right)}{L_{n_{j}+1} \prod_{k=j+1}^{m}\left(1+\frac{M_{n_{k}}}{L_{n_{k}}} \tan \left(\theta_{n_{k}-1}-\theta_{n_{k}}\right) \tan \left(\theta_{n_{k}}-\theta_{n_{k}+1}\right)\right) \prod_{l=n_{j}+1}^{n} \cos \left(\theta_{l}-\theta_{l+1}\right)} \\
= & \frac{v\left(\tan \left(\theta_{n_{j}}-\theta_{n_{j}+1}\right)-\frac{M_{n_{j}}}{L_{n_{j}}} \tan \left(\theta_{n_{j}-1}-\theta_{n_{j}}\right)\right)}{L_{n_{j}+1} \prod_{k=j}^{m}\left(1+\frac{M_{n_{k}}}{L_{n_{k}}} \tan \left(\theta_{n_{k}-1}-\theta_{n_{k}}\right) \tan \left(\theta_{n_{k}}-\theta_{n_{k}+1}\right)\right) \prod_{l=n_{j}+1}^{n} \cos \left(\theta_{l}-\theta_{l+1}\right)} \\
& j \in\{1, \ldots, m\}
\end{aligned}
$$


where $\theta_{n+1}=0$ and the generating input $v$ has been put into evidence. It is convenient to define the input of the $j$-th virtual wheel as

$$
\omega_{n_{j}} \triangleq \dot{\theta}_{n_{j}+1}
$$

so that the right side of eq. (42) will give the nonlinear state feedback that decides the steering angle of the $j$-th passive steering wheel. Clearly, this feedback constitutes the link between consecutive trains.

Using eq. (38), also the dynamic equation of all the orientation angles can be expressed as a linear homogeneous function of the generating input $v$ :

$$
\begin{aligned}
\dot{\theta}_{n_{j}-i} & =\frac{v \tan \left(\theta_{n_{j}-i-1}-\theta_{n_{j}-i}\right)}{L_{n_{j}-1} \prod_{k=j}^{m}\left(1+\frac{M_{n_{k}}}{L_{n_{k}}} \tan \left(\theta_{n_{k}-1}-\theta_{n_{k}}\right) \tan \left(\theta_{n_{k}}-\theta_{n_{k}+1}\right)\right) \prod_{l=n_{j}-i}^{n} \cos \left(\theta_{l}-\theta_{l+1}\right)} \\
\triangleq & v f_{n_{j}-i}\left(\underline{\theta}_{n_{j}-i-1}\right) \\
& j \in\{1, \ldots, m+1\}, \quad i \in\left\{0,1, \ldots, n_{j}-n_{j+1}-2\right\}, n_{m+1}=n .
\end{aligned}
$$

where

$$
\underline{\theta}_{i} \triangleq\left[\theta_{i}, \theta_{i+1}, \ldots, \theta_{n}\right]
$$

With these notations the dynamic of the general $n$-trailer system becomes:

$$
\begin{aligned}
\dot{x}_{n} & =v \\
\dot{\theta}_{n_{j}-i} & =v f_{n_{j}-i}\left(\underline{\theta}_{n_{j}-i-1}\right) \\
\dot{\theta}_{n_{j-1}+1} & =\omega_{j-1} \\
\dot{y}_{n} & =v f_{n+1}\left(\underline{\theta}_{n}\right)
\end{aligned}
$$

$j \in\{1, \ldots, m+1\}, i \in\left\{0,1, \ldots, n_{j}-n_{j+1}-2\right\}$, with the $\omega_{j}$ obtained via feedback from the state as in eq. (42). The configuration space is then:

$$
\mathbf{q}=\left[x_{n} \theta_{0} \theta_{1} \ldots \theta_{n} y_{n}\right]
$$

and we will consider the following domain for the change of coordinates:

$$
\begin{aligned}
& \mathcal{D}=\left\{\mathbf{q} \in R \times\left(S^{1}\right)^{n+1} \times R:\left|\theta_{n}\right|<\frac{\pi}{2}, \quad\left|\theta_{n_{j}-i-1}-\theta_{n_{j}-i}\right|<\frac{\pi}{2},\right. \\
& \left|\theta_{n_{j}}-\theta_{n_{j}+1}+\arctan \left(-\frac{M_{n_{j}}}{L_{n_{j}}} \tan \left(\theta_{n_{j}-1}-\theta_{n_{j}}\right)\right)\right|<\frac{\pi}{2}, \\
& \left.j \in\{1, \ldots, m+1\}, \quad i \in\left\{0,1, \ldots, n_{j}-n_{j+1}-2\right\}\right\}
\end{aligned}
$$

Basically, Prop. 1 is sufficient to cast the general n-trailer problem into an $m+2$ multichained form with $m+1$ chains corresponding to the $m+1$ steering trains identified above, all having the same generator. However, the solution proposed by [Tilbury et al. 1995], due to the more generality of the problem (all exogenous inputs), requires to consider virtual trailers to be attached in front of each of the existing steering trains in order to 'decouple' the 
dynamics of each train from the preceding ones, through a dynamic feedback from the state. In our case this virtual extensions are not needed, because the dynamics of the steering trains are already decoupled one from the other, i.e. each of the orientation angles $\theta_{i}$ depends only on what happens in front of it, except for the passive steering wheels that create a connection between two consecutive chains. Therefore, in the following, we will transform each of the steering trains into a 2-input chained form, all with the same generator.

Theorem 2 There exists a local diffeomorphism that converts the system (44) into the multichained form:

$$
\begin{aligned}
& \dot{z}_{g}=v \quad \dot{z}_{0}=u_{0} \quad \dot{z}_{n_{1}+1}=u_{1} \quad \ldots \dot{z}_{n_{m}+1}=u_{m} \\
& \dot{z}_{1}=v z_{0} \quad \dot{z}_{n_{1}+2}=v z_{n_{1}+1} \quad \dot{z}_{n_{m}+2}=v z_{n_{m}+1}
\end{aligned}
$$

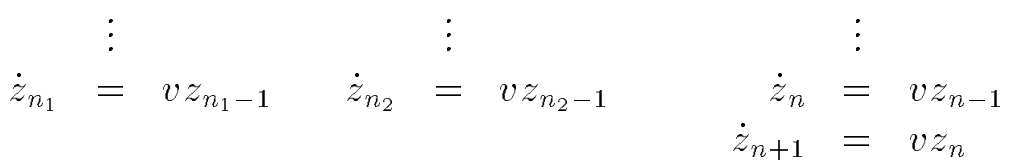

where $v$ and $u_{0}$ are exogenous inputs, while the $m$ functions $u_{i}, i=1, \ldots m$, are obtained via (nonlinear) feedback from the state.

Proof.

We can apply the algorithm of [Sørdalen 1993a] to each of the $m+1$ steering chains. For the last train, the chain will have an extra state (that will be called $z_{n+1}$ ) with respect to the other steering trains of the same length. The bottom of the chain is the second cartesian coordinate of the last trailer

$$
z_{n+1} \triangleq y_{n}
$$

Differentiating with respect to time, we get:

$$
\dot{y}_{n}=v \tan \theta_{n}=v f_{n+1}\left(\underline{\theta}_{n}\right) \triangleq v z_{n}
$$

and, differentiating again,

$$
\dot{z}_{n}=v \frac{\tan \left(\theta_{n-1}-\theta_{n}\right)}{L_{n} \cos ^{3} \theta_{n}}=v L_{\underline{f}_{n}} f_{n+1} \triangleq v z_{n-1}
$$

where we define:

$$
\underline{f}_{i} \triangleq\left[f_{i} f_{i+1} \ldots f_{n}\right]
$$

and $L_{f} h$ is the Lie derivative of $h$ along the vector $f$.

For the first $m$ steering trains we take as bottom of the corresponding chain the orientation angle of the trailer with off-hitching (the last of each steering train):

$$
z_{n_{j}} \triangleq \theta_{n_{j}} \quad j \in\{1,2, \ldots, m\}
$$

Using the generator $v$, the chained form is obtained from the bottom variables defined above using the relation

$$
\begin{array}{r}
\dot{z}_{n_{j}-i+1}=\dot{z}_{g} z_{n_{j}-i} \Rightarrow \quad z_{n_{j}-i}=\frac{\dot{z}_{n_{j}-i+1}}{\dot{z}_{g}} \\
j \in\{1,2, \ldots, m+1\} ; \quad i \in\left\{1,2, \ldots, n_{j}-n_{j-1}-1\right\}
\end{array}
$$


by means of a sequence of time differentiations. To have the same structure in all the $m+1$ chains despite the extra variable present in the last chain, we need to apply a 'cosmetic' change of index, calling $n_{m+1}=n+1$ (remember that, instead, we had defined before $\left.n_{m+1}=n\right)$. Now, for all the $m+1$ chains we have:

$$
\begin{aligned}
z_{n_{j}-1} & =f_{n_{j}}\left(\underline{\theta}_{n_{j}-1}\right) \\
z_{n_{j}-2} & =L_{\underline{f}_{n_{j}-1}} f_{n_{j}} \\
& \vdots \\
z_{n_{j}-i} & =L_{\underline{f}_{n_{j}-i+1}} L_{\underline{f}_{n_{j}-i+2}} \ldots L_{\underline{f}_{n_{j}-1}} f_{n_{j}} \\
& \vdots \\
z_{n_{j-1}+1} & =L_{\underline{f}_{n_{j-1}+2}} L_{\underline{f}_{n_{j-1}+3}} \ldots L_{\underline{f}_{n_{j}-2}} L_{\underline{f}_{n_{j}-1}} f_{n_{j}}
\end{aligned}
$$

with $j \in\{1,2, \ldots, m+1\}$ and $i \in\left\{1,2, \ldots, n_{j}-n_{j-1}-1\right\}$. The first variable of each chain is $z_{n_{j-1}+1}=z_{n_{j-1}+1}\left(\underline{\theta}_{n_{j-1}+1}\right)$ so, when we derive, also the input $\omega_{j-1}$ will appear into the expression. Therefore we define the new input $u_{j-1}$ as:

$$
u_{j-1} \triangleq \dot{z}_{n_{j-1}+1}=L_{\underline{f}_{n_{j-1}+1}} L_{\underline{f}_{n_{j-1}+2}} \ldots L_{\underline{f}_{n_{j}-2}} L_{\underline{f}_{n_{j}-1}} f_{n_{j}}
$$

where

$$
\underline{f}_{n_{j-1}+1}=\left[\omega_{j-1} f_{n_{j-1}+2} \ldots f_{n_{m}} \omega_{m} f_{n_{m}+2} \ldots f_{n+1}\right] \quad j \in\{1, \ldots m+1\}
$$

This marks the end of the chain and holds also for the first chain, the one depending on the real steering input $\omega_{0}$. So, for example, for the second-to-last ( $m$-th) chain, we will have

$$
\begin{aligned}
z_{n_{m}} & =\theta_{n_{m}} \\
z_{n_{m}-1} & =f_{n_{m}} \\
z_{n_{m}-2} & =L_{\underline{f}_{n_{m}-1}} f_{n_{m}}
\end{aligned}
$$

where

$$
\underline{f}_{n_{m}-1}=\left[f_{n_{m}-1} \omega_{m} f_{n_{m}+2} \ldots f_{n+1}\right]
$$

We know that, from the feedback law (43), the input is

$$
\omega_{m}=\omega_{m}\left(\underline{\theta}_{n_{m}-1}\right)
$$

and it bridges between the $m+1$-th and $m$-th chains. On the other hand, $\omega_{m}$ does not enter into the definition of the immediately following state $z_{n_{m}}$ (the bottom of the next chain), but only on the third one $z_{n_{m}-2}$. So the 'anomaly' introduced by the feedback in $\omega_{m}$ does not spoil the triangular structure of the change of base, since $\theta_{n_{m}-1}$ is already present also in $f_{n_{m}}$, i.e. in the second state of the new chain. This holds for all chains and we can write:

$$
\underline{f}_{n_{j}-i}=\underline{f}_{n_{j}-i}\left(\underline{\theta}_{n_{j}-i-1}\right)
$$


for $j \in\{1, \ldots m+1\}$ and $i \in\left\{0,1, \ldots n_{j}-n_{j-1}-1\right\}$.

If we call $\mathbf{z} \triangleq\left[z_{g} z_{0} z_{1} \ldots z_{n+1}\right]^{T}$ the new state vector in the chained form, then the transformation above calculated,

$$
\mathbf{z}=\Psi(\mathbf{q})
$$

is a diffeomorphism in $\mathcal{D}$. In fact, it is easily proven that the Jacobian $\frac{\partial \Psi}{\partial \mathbf{q}}$ is nonsingular in $\mathcal{D}$, since it is upper diagonal with nonnull diagonal elements in $\mathcal{D}$. The upper diagonal form of the Jacobian is a consequence of the aforementioned argument that each of the $z_{i}$ depends only on the orientation angles of the trailers up to the $i$-th one, not to what happens in front of it:

$$
z_{i}=z_{i}\left(\underline{\theta}_{i}\right) \quad i \in\{0,1, \ldots n\}
$$

which implies that

$$
\frac{\partial z_{i}}{\partial \theta_{i-k}}=0 \quad \text { if } k>0
$$

All the elements of the diagonal:

$$
\frac{\partial z_{g}}{\partial x_{n}}, \frac{\partial z_{0}}{\partial \theta_{0}}, \frac{\partial z_{1}}{\partial \theta_{1}}, \ldots, \frac{\partial z_{n_{j}}}{\partial \theta_{n_{j}}}, \frac{\partial z_{n_{j}+1}}{\partial \theta_{n_{j}+1}} \ldots \frac{\partial z_{n+1}}{\partial y_{n}}
$$

are certainly nonzero. In particular, for the last state $z_{n_{j}} j \in\{1, \ldots m+1\}$ of each chain and for the generator $z_{g}$, the corresponding diagonal element will be 1 , while for the remaining states $z_{n_{j}-i} j \in\{1, \ldots m+1\} \quad i \in\left\{1, \ldots n_{j}-n_{j-1}-1\right\}$ it will be proportional to the term $\frac{1}{\cos ^{2}\left(\theta_{n_{j}-i}-\theta_{n_{j}-i+1}\right)}$ that corresponds to the derivative of a tangent function. This assures the nonsingularity of the Jacobian matrix in the domain $\mathcal{D}$.

Also the $m+2$ input transformation can be easily shown to be well-defined in $\mathcal{D}$. We saw in eq. (39) that the generating input $v$ (for $M_{0}=0$ ) can be obtained from $v_{0}$. With regard to the virtual and real steering inputs, eq. (46) says that the corresponding input in the chained form $u_{j}, j \in\{1, \ldots m\}$, is a function of the original inputs up to the $j$-th:

$$
u_{j}=u_{j}\left(\omega_{j}, \omega_{j+1}, \ldots, \omega_{m}, \underline{\theta}_{n_{j}-1}\right) .
$$

Therefore also the input transformation is a local diffeomorphism.

According to eq. (43), the input $u_{j-1}$ can be thought of as state feedback from the existing state:

$$
u_{j-1}=g\left(w_{j-1}, \underline{\theta}_{n_{j-1}+1}\right)=g\left(\underline{\theta}_{n_{j-1}}\right)
$$

where now $\omega_{j}$ and $\theta_{j}$ can be considered as obtained from the inverse diffeomorphism of the above described state and input transformations in a domain containing the origin.

Unlike the standard $n$-trailer case, this transformation is not very much useful in practice: in fact, trying to squeeze down to 'euclidean' the system along the regular parts of the chains results into an 'explosion' of the expression of the nonlinear feedback for the virtual wheels which becomes dependent on partial derivatives of the whole state. Attaining an explicit expression for the feedback in the new basis is quite prohibitive also for low-dimensional cases, because it requires to have an explicit expression for the inverse of the diffeomorphism $(47)$. 
The chained form is known to be the dual canonical form of the so-called Goursat normal form for higher order contact manifolds, see [Bryant et al. 1991]. The fact that kingpin hitches spoils the transformation can be seen also from the derivative flag (40). The double loss of rank of the derived codistribution in correspondence to each off-axle hooking implies that the basis used in the Goursat normal form theorem is not adapted to the derivative flag of $I$ because one has to look for $m$ integrable functions orthogonal to $I$ (other that the usual $\pi \neq 0 \bmod I$ ) one for each kingpin hitch. In the same spirit as [Tilbury et al. 1995], it is possible to add virtual one-forms, corresponding to the virtual steering wheels, i.e. first order prolongations to the system. The constraint $\omega^{j}$ on the $j$-th virtual steering wheel has the expression:

$$
\omega^{j}=\sin \theta_{\gamma_{j}} d x_{\gamma_{j}}-\cos \theta_{\gamma_{j}} d y_{\gamma_{j}}=0
$$

where $\left(x_{\gamma_{j}}, y_{\gamma_{j}}\right)$ are the cartesian coordinates of the kingpin hitch point and $\theta_{\gamma_{j}}$ is the orientation angle of the virtual wheel. The corresponding dynamic equations can be easily calculated from the geometry (see Fig. 2) and from the expression for the virtual feedback calculated in this Section. It can be seen that, although the virtual constraints 'regularize' the derivative flag of the original system, the augmented system is still not regular. Adding sufficient many dynamic prolongations (which have the meaning of other virtual trailers added in front of the virtual steering wheels, see [Tilbury et al. 1995]), it is probably possible to achieve a derivative flag with towers that decrease regularly (see [Bushnell et al. 1993] for details). However, the bottom line is that the new virtual steering angle, which is now the derivative of some order of the virtual steering angle $\gamma_{j}$, is still going to have an expression which is a function of the original state. In this augmented basis, (not yet transformed into chained form) prolonging the virtual inputs means 'prolonging the feedback law' i.e. adding terms to the virtual feedback (43) such that its new expression is a function of all the orientation angle of the real system standing in front of the corresponding kingpin hitch from which the prolongation originates (and not a simple one....).

\section{Conclusion}

The kinematic analysis of the so-called $n$-trailer system is usually limited to the special case of axle-to-axle hitching between trailers. This is done not only for sake of simplicity, but also because such a model presents a number of interesting properties which are (relatively) easy to verify, like nonlinear controllability, conversion into chained form or differential flatness, all due to the nonholonomic nature of the system. For the more general configuration considered in this paper, which includes also more realistic off-axle connections between trailers, it is shown how to verify or interpret some of these properties normally used in the standard configuration.

\section{References}

[Abraham et al. 1983] ABRAHAM R., MARSDEN J.E. and RATIU T., 1983, Manifolds, tensor analysis and applications (Springer-Verlag, 2nd ed.). 
[Alexander and Maddox 1988] ALEXANDER J.C. and MADDOX J.H., 1988, On the maneuvering of vehicles, SIAM Journal of Applied Mathematics,48,38-51.

[Altafini 1999] ALTAFINI C., 1999, A path tracking criterion for an LHD articulated vehicle, International Journal of Robotics Research,18,435-441.

[Bloch et al. 1992] BLOCK A., REYHANOGLU M. and MCCLAMROCH N.H., 1992, Control and stabilization of nonholonomic dynamical systems, IEEE Trans. on Automatic Control, 37,1746-1757.

[Bryant et al. 1991] BRYANT R.L., CHERN S.S., GARDNER R.B., GOLDSCHMIDT H.L. and GRIFFITHS P.A., 1991, Exterior Differential Systems (Springer-Verlag).

[Bushnell et al. 1993] BUSHNELL L.G., TILBURY D. and SASTRY S., 1993, Steering three-input chained form nonholonomic systems using sinusoids: the fire truck example, Proc. of the 2nd European Control Conference, Groningen, The Netherlands, pp.1432-1437.

[Bushnell et al. 1993] BUSHNELL L.G., TILBURY D. and SASTRY S., 1993, Extended Goursat normal form with applications to nonholonomic motion planning, Proc. of the 32nd Conference on Decision and Control, San Antonio, TX, pp.3447-3452.

[Fliess et al. 1995] FLIESS M., LEVINE J., MARTIN P. and ROUCHON P., 1995, Flatness and defect of nonlinear systems: introductory theory and examples, Int. Journal of Control,61,1327-1361.

[Hermann and Krener 1977] HERMANN R. and KRENER A..J., 1977, Nonlinear controllability and observability, IEEE Trans. on Automatic Control, 22,728-740.

[Isirori 1995] ISIDORI A., 1995, Nonlinear Control Systems (Springer-Verlag, 3rd ed.).

[Jean 1996] JEAN F., 1996, The car with n trailers: characterization of the singular configuration, EISAM: control optimization and calculus of variations, 1,241-266.

[Kolmanovsky and McClamroch 1995] KOLMANOVSKY I. and MCCLAMROCH N.H., 1995, Developments in nonholonomic control problems, IEEE Control System Magazine, December, pp.20-36.

[Laumond 1990] LAUMOND J.P., 1990, Nonholonomic motion planning versus controllability via the multibody car system example, Dep. of Computer Science, Stanford University, STAN-CS-90-1345.

[Laumond 1993a] LAUMOND J.P., 1993, Singularities and topological aspects in nonholonomic motion planning, in Z. Li and J.F. Canny (eds.), Nonholonomic Motion Planning (Kluwer Academic, Boston, MA).

[Laumond 1993b] LAUMOND J.P., 1993, Controllability of a multibody mobile robot, IEEE Trans. on Robotics and Automation,9,755-763.

[Murray and Sastry 1993] MURRAY R. and SASTRY S., 1993, Nonholonomic motion planning: steering with sinusoids, IEEE Trans. on Automatic Control, 38,700-716.

[Nijmeijer and van der Shaft 1990], NIJMEIJER H. and VAN DER SHAFT A.J., 1990, Nonlinear Dynamical Control Systems (Springer-Verlag). 
[Pappas et al. 1998] PAPPAS G.J., LYGEROS J., TILBURY D. and SASTRY S., 1998, Exterior Differential Systems in Control and Robotics, in J. Baillieul, S. Sastry and H. Sussmann (eds.) Essays on Mathematical Robotics (IMA Volumes in Mathematics and its Applications, Springer-Verlag).

[Rouchon et al. 1993] ROUCHON P., FLIESS M., LEVINE J. and MARTIN P., 1993, Flatness, and motion planning: the car with $n$ trailers, Proc. 2nd European Control Conference, Groningen, The Netherlands, pp.1518-1522.

[Samson 1995] SAMSON C., 1995, Control of chained systems: application to path-following and time-varying point stabilization of mobile robots, IEEE Trans. on Automatic Control,40,64-77.

[Sørdalen 1993a] SøRDALEN O.J., 1993, Conversion of the kinematics of a car with $n$ trailers into chained form, Proc. IEEE Int. Conf. on Robotics and Automation, Atlanta, Georgia, pp.382-387.

[Sørdalen 1993b] SøRDALEN O.J., 1993, Exponential stabilization of a car with $n$ trailers, Proc. IEEE 32nd Conf. on Decision and Control, San Antonio, Texas, pp.978-983.

[Sørdalen and Egeland 1995] SøRDALEN O.J. and EGELAND O., 1995, Exponential stabilization of nonholonomic chained systems, IEEE Trans. on Automatic Control,40,35-49.

[Spivak 1979] SPIVAK M., 1979, A comprehensive introduction to Differential Geometry, vol I (Publish or Perish, Houston, TX).

[Tilbury et al. 1995] TILBURY D., MURRAY R. and SASTRY S., 1995, Trajectory generation for the N-trailer problem using Goursat normal form, IEEE Trans. on Automatic Control,40,802-819.

[Tilbury et al. 1995] TILBURY D., SØRDALEN O.J., BUSHNELL L. and SASTRY S., 1995, A multisteering trailer system: conversion into chained form using dynamic feedback, IEEE Trans. on Robotics and Automation,6,807-818. 